GUEST EDITORIAL

\title{
Uncertainty quantification for engineering design
}

\author{
ROGER GHANEM $^{1}$ AND XIAOPING DU ${ }^{2}$ \\ ${ }^{1}$ Department of Civil and Environmental Engineering, University of Southern California, Los Angeles, California, USA \\ ${ }^{2}$ Department of Mechanical and Aerospace Engineering, Missouri University of Science and Technology, Rolla, Missouri, USA
}

Engineering design is generally predicated on a presumed behavior of a given system in response to a specified range of operational conditions. With improved sensing, control, and adaptation schemes, both external and internal conditions are characterized with increasing accuracy, permitting safer operation closer to the failure envelope. These technological and scientific advances notwithstanding, discrepancies remain between anticipated and actual behaviors of most engineered systems, often becoming significant as instabilities or failure are approached. The ability to understand, analyze, and characterize the sources and the impact of these errors will have important ramifications on the economy, performance, and safety of these systems.

Recent advances on the topic of uncertainty quantification have enabled the closer integration of data-driven and modeldriven paradigms for parameter characterization and performance prediction of many systems of interest in science and engineering. The impact of these capabilities on engineering design are just beginning to be felt with significant implications on the interplay between performance, efficiency, and risk of both specific products and the design process as a whole.
This special issue of AI EDAM brings together a sampling of contributions from the forefront of research in uncertainty quantification for engineering design. As demonstrated by the breadth of these contributions, improvements of the design process can be achieved through a range of interventions, including system-level modeling and planning under uncertainty; integration of data acquisition into system design and performance; conceptual and mathematical perspectives on representing uncertain parameters and objectives; efficient statistical computing; and data, model, and decision fusion through hybrid algorithms. The range of applications is also, as expected, very broad, encompassing supply-chain systems, manufacturing, aerospace, automotive, natural resources, and oil and gas.

\section{ACKNOWLEDGMENTS}

We thank Professor Yan Jin for his foresight and invitation for us to realize this Special Issue. We also thank Professor Amaresh Chakrabarti for his expert editorial opinion and for working with us through the final stages of production.

Reprint requests to: Roger Ghanem, Department of Civil and Environmental Engineering, 210 Kap Hall, University of Southern California, 3610 South Vermont, Los Angeles, CA 90089, USA. E-mail: ghanem@usc.edu 